Series A

I. MATHEMATICA

449

\title{
REMOVABILITY THEOREMS FOR QUASICONFORMAL MAPPINGS
}

BY

SEPPO RICKMAN

H E L S I N K I 1969

S U O M A L A I NEN T I E D E A K A T E M I A

doi:10.5186/aasfm.1969.449 
Communicated 9 May 1969 by Oldi Lento and K. I. Virtanen 


\section{Removability theorems for quasiconformal mappings}

1. Introduction. In this paper we shall study the following removability question: Let $D$ and $D^{\prime}$ be domains in the euclidean $n$-space $R^{n}, n \geq 2$, let $E \subset D$ be closed in $D$, and let $f: D \rightarrow D^{\prime}$ be a homeomorphism which is locally $K$-quasiconformal in $D-E$ for some $K$, which means that for every $x \in D-E$ there is a connected neighborhood $U$ of $x$ such that $f \mid U$ is a $K$-quasiconformal mapping [8, p. 20]. We ask for conditions on $E$ and on the restriction $f \mid E$ which imply the quasiconformality of $f$. A special case for $n=2$ of this situation is considered in [5, Theorem 3] which implies that $f$ is quasiconformal if $E$ is a Jordan curve and if $f|E=g| E$ for some quasiconformal mapping $g$ of a domain $G \supset E$.

The set $E$ is called an exceptional set if $f$ is always a $K$-quasiconformal mapping. One of the main results which give conditions for the exceptionality is that the set $E$ is exceptional if $E$ is of $\sigma$-finite $(n-1)$-dimensional Hausdorff measure [9, 35.1], [3, Corollary 5]; for the case $n=2$ see also [7], [1], and [4, Satz V.3.2]. We shall give answers to the given problem in the other direction. It turns out (Theorem 1) that the condition mentioned above, namely the existence of a quasiconformal mapping $g$ of a domain $G \supset E$ such that $f|E=g| E$, implies the quasiconformality of $f$ even if no further assumptions are made on $E$. We shall also in Theorem 2 establish another form where the assumption on the restriction $f \mid E$ is weakened but $E$ is assumed to be connected or locally connected. In these results the maximal dilatation of $f$ is in general greater than $K$.

2. Notation. Throughout this paper $D$ and $D^{\prime}$ are domains in $R^{n}$ and $n \geq 2$. If $A, B \subset R^{n}, d(A, B)$ denotes the euclidean distance between $\bar{A}$ and $B$. For $x \in R^{n}$ and $r>0$ we set $B^{n}(x, r)=\left\{y \in R^{n} \mid\right.$ $|y-x|<r\}$ and $S^{n-1}(x, r)=\left\{y \in R^{n} \mid y-x=r\right\}$. We also use the abbreviations $B^{n}(r)=B^{n}(0, r)$ and $S^{n-1}(r)=S^{n-1}(0, r)$. If $f: D \rightarrow D^{\prime}$ is a homeomorphism, if $x \in D$, and if $0<r<d(x, \partial D)$, we set

$$
\begin{gathered}
L(x, f, r)=\sup _{|y-x|=r}|f(y)-f(x)|, \\
l(x, f, r)=\inf _{|y-x|=r}|f(y)-f(x)| .
\end{gathered}
$$

The linear dilatation of $f$ at $x$ is 


$$
H(x, f)=\limsup _{r \rightarrow 0} \frac{L(x, f, r)}{l(x, f, r)} .
$$

The $k$-dimensional Lebesgue measure is denoted by $m_{k}$. The $(n-1)$ dimensional measure of the unit sphere $S^{n-1}(1)$ is $\omega_{n-1}$.

3. We start with a simple distortion result for quasiconformal mappings. Let $v$ be an increasing function of the interval $[1, \infty)$ into itself and let $\varphi: A \rightarrow R^{n}, A \subset R^{n}$, be an injective mapping. We say that $\varphi$ has local $v$-bounded distortion if for every $x \in A$ there is an $s>0$ such that $x_{1}, x_{2} \in A$ and $0<\left|x-x_{2}\right| \leq\left|x-x_{1}\right|<s$ imply

$$
\frac{\left|\varphi\left(x_{1}\right)-\varphi(x)\right|}{\left|\varphi\left(x_{2}\right)-\varphi(x)\right|} \leq v\left(\frac{\left|x_{1}-x\right|}{\left|x_{2}-x\right|}\right) .
$$

Lemma 1. Let $f: D \rightarrow D^{\prime}$ be a K-quasiconformal mapping. Then there exists an increasing function $v:[1, \infty) \rightarrow[1, \infty)$ depending only on $n$ and $K$ such that $f$ has local v-bounded distortion.

Proof. Assume $x \in D$. Choose $s>0$ such that $f \bar{B}^{n}(x, s) \subset$ $\bar{B}^{n}(f(x), t) \subset D^{\prime} \quad$ for some $\quad t$. Let $\quad 0<\left|x_{2}-x\right| \leq\left|x_{1}-x\right|<s$ and set $\alpha_{i}=\left|f\left(x_{i}\right)-f(x)\right|, i=1,2$. Assume $\alpha_{2}<\alpha_{1}$ and let $\Gamma^{\prime}$ be the family of curves which join the boundary components of the ring $A^{\prime}=$ $\left\{y\left|\alpha_{2}<\right| y-f(x) \mid<\alpha_{1}\right\}$ in $A^{\prime}$. Then the $n$-modulus $M\left(\Gamma^{\prime}\right)$ of $\Gamma^{\prime}$ equals $\omega_{n-1} /\left(\log \left(\alpha_{1} / \alpha_{2}\right)\right)^{n-1}[8$, p. 5, 7]. For the $n$-modulus of the curve family $\Gamma=f^{-1} \Gamma^{\prime}=\left\{f^{-1} \circ \gamma^{\prime} \mid \gamma^{\prime} \in \Gamma^{\prime}\right\} \quad$ we get by $[9,11.7]$ (see also [2, Theorem 4]) the estimate

$$
M(\Gamma) \geq \varkappa_{n}\left(\frac{\left|x_{1}-x\right|}{\left|x_{2}-x\right|}\right)
$$

where $\varkappa_{n}:(0, \infty) \rightarrow(0, \infty)$ is a decreasing function which depends only on $n$. Since $f$ is $K$-quasiconformal, $M(\Gamma) \leq K M\left(\Gamma^{\prime}\right)$. Hence

$$
\frac{\left|f\left(x_{1}\right)-f(x)\right|}{\left|f\left(x_{2}\right)-f(x)\right|} \leq \exp \left(\left(\frac{K \omega_{n-1}}{\varkappa_{n}\left(\frac{\left|x_{1}-x\right|}{\left|x_{2}-x\right|}\right)}\right)^{1 /(n-1)}\right)=v\left(\frac{\left|x_{1}-x\right|}{\left|x_{2}-x\right|}\right),
$$

and the lemma is proved.

The main step is the following lemma (cf. [5, Lemma 3]).

Lemma 2. Let $E \subset D$ be closed in $D$ and let $f: D \rightarrow D^{\prime}$ be a homeomorphism which is locally $K$-quasiconformal in $D-E$ and such that $f \mid E$ has local $v$-bounded distortion for some $v$. Let $E_{0}$ be the set of points $x \in E$ such that for every integer $j$ there exists an integer $k \geq j$ such that $\left(B^{n}(x, 1 / k)\right.$ $\left.-B^{n}(x, 1 / 2 k)\right) \cap E=\emptyset$. Then 
(a) $m_{n}\left(E_{0}\right)=0$.

(b) There exists a $c<\infty$, depending only on $n, K$, and $v$, such that $H(x, f)<c$ if $x \in D-E_{0}$.

Proof. Since no point of $E_{0}$ is a point of outer density for $E_{0}, m_{n}\left(E_{0}\right)=0$ $[6$, p. 129].

To prove (b) it suffices by $[9,34.2]$ to show that a uniform bound exists for $H(x, f)$ for points $x \in E-E_{0}$. Let $x_{0} \in E-E_{0}$. By performing similarity transformations we may assume that $x_{0}=f\left(x_{0}\right)=0$. There exists an integer $k_{0}$ such that $\left(B^{n}(1 / k)-B^{n}(1 / 2 k)\right) \cap E \neq \emptyset$ for $k \geq k_{0}$. Since $f \mid E$ has local $v$-bounded distortion, there exists an $s>0$ such that if $x_{1}, x_{2} \in E$ and if $0<\left|x_{2}\right| \leq\left|x_{1}\right|<s$, then

$$
\frac{\left|f\left(x_{1}\right)\right|}{\left|f\left(x_{2}\right)\right|} \leq v\left(\frac{\left|x_{1}\right|}{\left|x_{2}\right|}\right) \text {. }
$$

Now let $r$ be such that $0<r<\min \left(d(0, \partial D), s, 1 / k_{0}\right) / 8$ and such that $\bar{B}^{n}(L(0, f, r)) \subset D^{\prime}$. We set

$$
\begin{aligned}
L_{r} & =L(0, f, r), l_{r}=l(0, f, r), \\
A_{1} & =\{x|r<| x \mid<2 r\}, A_{2}=\{x|r / 2<| x \mid<r\}, \\
H_{1} & =\{x|2 r<| x \mid<8 r\}, H_{2}=\{x|r / 8<| x \mid<r / 2\}, \\
F_{i} & =\bar{A}_{i} \cup \bar{H}_{i}, \quad i=1,2, \\
r_{1} & =\sup _{x \in E \cap F_{1}}|f(x)|, \quad r_{2}=\inf _{x \in E \cap F_{2}}|f(x)| .
\end{aligned}
$$

We shall make use of the fact that the sets $\bar{A}_{1}-f^{-1} \bar{B}^{n}\left(r_{1}\right)$ and $\bar{A}_{2} \cap f^{-1} B^{n}\left(r_{2}\right)$ do not meet $E$.

Assume $L_{r}>r_{1}$ and let $z \in f S^{n-1}(r)$ be such that $|z|=L_{r}$. There exists $\tau_{1}>1$ such that the line segment $J=\left\{\tau_{z}: 1 \leq \tau \leq \tau_{1}\right\}$ is contained in $f \bar{A}_{1}$ and such that $\tau_{1} z \in f S^{n-1}(2 r)$. Assume $r_{1}<\sigma<L_{r}$ and let $\Gamma$ be the family of curves which join $f^{-1} J$ and $f^{-1} S^{n-1}(\sigma)$ in $\bar{A}_{1}$ $f^{-1} B^{n}(\sigma)$.

Next we derive a positive lower bound for the $n$-modulus $M(\Gamma)$ of $\Gamma$. Let $r<t<2 r$ and set $S=S^{n-1}(t)$. Then $S \cap f^{-1} J \neq \varnothing$. We show that also $S \cap f^{-1} S^{n-1}(\sigma) \neq \varnothing$ holds. To prove this we first note that $\bar{H}_{1} \cap E \neq \varnothing$. There is therefore a point $u \in \bar{B}^{n}\left(r_{1}\right) \cap f \bar{H}_{1}$. The line segment $\{\lambda u \mid 0<\lambda<1\}$ meets $f S$. The assertion then follows from the fact that $f S$ has points in both components of the complement of $S^{n-1}(\sigma)$. We now choose a point $y \in S \cap f^{-1} J$. Since $y$ does not belong to the nonempty closed set $S \cap f^{-1} S^{n-1}(\sigma)$, there exists an open half space $M$ such that $y \in M, M \cap S \subset S-f^{-1} S^{n-1}(\sigma)$, and $\bar{M} \cap S \cap f^{-1} S^{n-1}(\sigma)$ $\neq \varnothing$. Denote by $\Gamma_{t}$ the family of curves $\gamma \in \Gamma$ which lie in $\bar{M} \cap S$. 
For the $n$-modulus $M_{n}^{S}\left(\Gamma_{t}\right)$ of $\Gamma_{t}$ with respect to $S$ the estimate $M_{n}^{S}\left(\Gamma_{t}\right)$ $\geq b_{n} / t$ holds where $b_{n}>0$ is a constant which depends only on $n[9$, 10.2]. If $\varrho: R^{n} \rightarrow[0, \infty]$ is a Borel function such that

$$
\int_{\gamma} \varrho d s \geq 1
$$

for every rectifiable $\gamma \in \Gamma$, we have

$$
\int_{S} \varrho^{n} d m_{n-1} \geq M_{n}^{S}\left(\Gamma_{t}\right)
$$

by definition. Hence

$$
\begin{aligned}
\int \varrho^{n} d m_{n} \geq & \int_{A_{1}} \varrho^{n} d m_{n}=\int_{r}^{2 r}\left(\int_{S^{n-1}(t)} \varrho^{n} d m_{n-1}\right) d t \\
& \geq \int_{r}^{2 r} \frac{b_{n}}{t} d t=b_{n} \log 2 .
\end{aligned}
$$

This gives $M(\Gamma) \geq b_{n} \log 2>0$.

On the other hand, the ring $B^{n}\left(L_{r}\right)-\bar{B}^{n}(\sigma)$ separates $J$ and $S^{n-1}(\sigma)$. Consequently, $M(f \Gamma) \leq \omega_{n-1} /\left(\log \left(L_{r} / \sigma\right)\right)^{n-1} \quad[8$, p. 7] where $f \Gamma=\{f \circ \gamma \mid$ $\gamma \in \Gamma\}$. Let $D_{1}$ be the component of $D-E$ which contains $f^{-1} J$. Then every curve of $\Gamma$ lies in $D_{1}$. Since $f \mid D-E$ is locally $K$-quasiconformal, $f \mid D_{1}$ is $K$-quasiconformal, and we have $M(\Gamma) \leq K M(f \Gamma)$. This gives $b_{n} \log 2 \leq K \omega_{n-1} /\left(\log \left(L_{r} / \sigma\right)\right)^{n-1}$. Hence

$$
\frac{L_{r}}{r_{1}} \leq \exp \left(\left(\frac{K \omega_{n-1}}{b_{n} \log 2}\right)^{1 /(n-1)}\right)=a_{n} .
$$

Similarly one proves $l_{r} / r_{2} \geq a_{n}^{-1}$.

Let $x_{i} \in E \cap F_{i}$ be such that $\left|f\left(x_{i}\right)\right|=r_{i}, i=1,2$. Then $\left|x_{1}\right| /\left|x_{2}\right|$ $\leq 64$. Finally we obtain the estimate

$$
\frac{L_{r}}{l_{r}} \leq a_{n}^{2} v(64)
$$

which proves (b).

Theorem 1. Let $E \subset D$ be closed in $D$ and let $f: D \rightarrow D^{\prime}$ be a homeomorphism which is locally $K$-quasiconformal in $D-E$ for some $K$. Suppose that there exists a quasiconformal mapping $g$ of a domain $G, E \subset G \subset D$, such that $g|E=f| E$. Then $f$ is quasiconformal. 
Proof. By Lemma 1, $f \mid E$ has local $v$-bounded distortion where $v$ depends only on $n$ and on the maximal dilatation $K(g)$ of $g$. By Lemma 2 , there exists a set $E_{0} \subset E$ of measure zero such that $H(x, f)<c<\infty$ for $x \in D-E_{0}$ where $c$ depends only on $n, K$, and $K(g)$. By an $n$ dimensional version of [8, Lemma 6.3], $f$ is differentiable almost everywhere. If $f$ is differentiable at $x \in D-E_{0},\left|f^{\prime}(x)\right|^{n} \leq c^{n-1}|J(x, f)|$ where $f^{\prime}(x)$ is the derivative and $J(x, f)$ the Jacobian of $f$ at $x$. We shall show that $f$ is ACL $[8$, p. 15]. The quasiconformality of $f$ then follows from an $n$-dimensional version of [8, Theorem 6.11].

It suffices to prove that $f$ is ACL in $G$. To show this, let $Q$ be an open $n$-interval such that $\bar{Q} \subset G$. Let $P: R^{n} \rightarrow R^{n-1}$ be the orthogonal projection. For each Borel set $A \subset P Q$ we set $Z_{A}=Q \cap P^{-1} A$ and $\psi(A)=m_{n}\left(f Z_{A}\right)$. By Lebesgue's theorem, the set function $\psi$ has a finite derivative $\psi^{\prime}(y)$ for almost every $y \in P Q$. Furthermore, $g$ is absolutely continuous on $Z_{y}$ and $m_{1}\left(E_{0} \cap Z_{y}\right)=0$ for almost every $y \in P Q$. Fix $y \in P Q$ such that all these three conditions are satisfied. By symmetry, it is sufficient to prove that $f$ is absolutely continuous on $\bar{Z}_{y}$.

Let $F$ be a compact subset of $Z_{y}$. Since $g$ is absolutely continuous on $Z_{y}$, since $g\left|E_{0}=f\right| E_{0}$, and since $m_{1}\left(E_{0} \cap F\right)=0$, we have $\Lambda_{1}\left(f\left(E_{0}\right.\right.$ $\cap F))=0 \quad$ where $A_{1}$ is the 1-dimensional Hausdorff measure. Hence $\Lambda_{1}(f F)=\Lambda_{1}\left(f\left(F-E_{0}\right)\right)$. Let $k_{0}$ be an integer such that $0<1 / k_{0}<$ $d(F, \partial Q)$. For each integer $k \geq k_{0}$ we define the set $F_{k}$ of points $x \in F$ such that $0<r<1 / k$ implies $L(x, f, r) \leq c l(x, f, r)$. For every $k \geq k_{0}$ $F_{k}$ is compact and $F_{k} \subset F_{k+1}$. Since $H(x, f)<c$ for $x \in F-E_{0}$, we have

$$
F-E_{0} \subset \bigcup_{k=k_{0}}^{\infty} F_{k}=\dot{F}
$$

and one can prove the inequality (see $[9,(31.3)]$ and $[1$, p. 10])

$$
\Lambda_{1}(f \hat{F})^{n} \leq \alpha c^{n} \psi^{\prime}(y) m_{1}(F)^{n-1}
$$

where $\alpha<\infty$ is a constant which depends only on $n$. Consequently, also $\Lambda_{1}(f F)^{n}$ has the right hand side of (1) as an upper bound. After this a simple limiting process shows that $f$ is absolutely continuous on $\bar{Z}_{y}$. The theorem is proved.

Theorem 2. Let $E \subset D$ be connected or locally connected and closed in $D$. Let $f: D \rightarrow D^{\prime}$ be a homeomorphism which is locally $K$-quasiconformal in $D-E$ for some $K$. Suppose further that $f \mid E$ has local $v$-bounded distortion for some $v$. Then $f$ is quasiconformal.

Proof. The set $E_{0} \subset E$ defined in Lemma 2 consists in this case of isolated points only, and $D-E_{0}$ is a domain. By (b) in Lemma 2 and by $[9,34.1] f \mid D-E_{0}$ is quasiconformal. But $E_{0}$ is removable $[9,17.3]$, and the theorem is proved. 
Remark. If $\varphi$ has local $v$-bounded distortion for some $v$, it does not necessarily follow that $\varphi$ is a restriction of a quasiconformal mapping. This is shown by an $n$-dimensional version of the example presented in [5, p. 388]. Hence the condition on $f \mid E$ is in this sense weaker in Theorem 2 than in Theorem 1.

\section{University of Helsinki}

Helsinki, Finland

\section{References}

1. Gehring, F. W.: The definitions and exceptional sets for quasiconformal mappings. - Ann. Acad. Sci. Fenn. A I 281 (1960), 1-28.

2. -»- Symmetrization of rings in space. - Trans. Amer. Math. Soc. 101 (1961), $499-519$.

3. - - Rings and quasiconformal mappings in space. - Trans. Amer. Math. Soc. 103 (1962), 353-393.

4. Lehto, O. and K. I. Virtanen: Quasikonforme Abbildungen. - Springer-Verlag, 1965.

5. Rickman, S.: Quasiconformally equivalent curves. - Duke Math. J. 36 (1969), $387-400$.

6. SAKs, S.: Theory of the integral. - Hafner Publishing Company, 1937.

7. Strebel, K.: On the maximal dilation of quasiconformal mappings. - Proc. Amer. Math. Soc. 6 (1955), 903-909.

8. VäISÄLÄ, J.: On quasiconformal mappings in space. - Ann. Acad. Sci. Fenn. A I 298 (1961), $1-36$.

9. -»- Lectures on $n$-dimensional quasiconformal mappings. - Van Nostrand, to appear. 